\title{
Choice of reconstructed tissue properties affects interpretation of lung EIT images
}

\author{
Bartłomiej Grychtol ${ }^{1}$ and Andy Adler ${ }^{2}$ \\ ${ }^{1}$ Department of Medical Physics in Radiology, German Cancer Research Centre \\ (DKFZ), 69120 Heidelberg, Germany \\ ${ }^{2}$ Systems and Computer Engineering, Carleton University, Ottawa, ON K1S 5B6, \\ Canada \\ E-mail: adler@sce.carleton.ca
}

\begin{abstract}
Electrical impedance tomography (EIT) estimates an image of change in electrical properties within a body from stimulations and measurements at surface electrodes. There is significant interest in EIT as a tool to monitor and guide ventilation therapy in mechanically ventilated patients. In lung EIT, the EIT inverse problem is commonly linearised and only changes in electrical properties are reconstructed. Early algorithms reconstructed changes in resistivity, while most recent work using the finite element method reconstructs conductivity. Recently, we demonstrated that EIT images of ventilation can be misleading if the electrical contrasts within the thorax are not taken into account during the image reconstruction process. In this paper, we explore the effect of the choice of the reconstructed electrical properties (resistivity or conductivity) on the resulting EIT images. We show in simulation and experimental data that EIT images reconstructed with the same algorithm but with different parametrisations lead to large and clinically significant differences in the resulting images, which persist even after attempts to eliminate the impact of the parameter choice by recovering volume changes from the EIT images. Since there is no consensus among the most popular reconstruction algorithms and devices regarding the parametrisation, this finding has implications for potential clinical use of EIT. We propose a program of research to develop reconstruction techniques that account for both the relationship between air volume and electrical properties of the lung and artefacts introduced by the linearisation.
\end{abstract}




\section{Introduction}

Electrical Impedance Tomography (EIT) calculates images of the change in conductivity distribution within a body from electrical stimulation and measurement on the body surface. Perhaps the most promising application of EIT is for monitoring the lungs of patients receiving ventilatory therapy (Adler et al. 2012). In such patients the distribution of inspired air may be very non-uniform, and EIT monitoring is useful to determine the effect of clinical interventions on patient ventilation (Victorino et al. 2004). Thus, the interest in EIT is driven by its potential to manage and optimize patient specific ventilation settings (Wolf et al. 2013).

Virtually all experimental and clinical EIT research has used time difference imaging, in which an image of the change in tissue properties is calculated from the change in measured EIT data. Changes are defined between the current instant (with a distribution of tissue properties $\mathbf{p}$ and EIT frame data $\mathbf{v}$ ) and a reference instant, at which time the patient's physiology is assumed to be stable (with tissue properties $\mathbf{p}_{0}$ and EIT frame data $\mathbf{v}_{0}$ ). Thus time-difference EIT reconstructs a difference in tissue properties $\left(\Delta \mathbf{p}=\mathbf{p}-\mathbf{p}_{0} \triangleq \mathbf{m}\right)$ from changes in measured data $\left(\Delta \mathbf{v}=\mathbf{v}-\mathbf{v}_{0} \triangleq \mathbf{d}\right)$. $\Delta \mathbf{v}$ is often normalized by division with $\mathbf{v}_{0}$; we refer to this case as normalized timedifference EIT. Time difference image reconstruction must assume a starting value of tissue properties, $\mathbf{p}_{0}$, which, in this paper, we call the "background properties".

Time difference EIT offers the advantage of being much less sensitive to uncertainties in electronic gain, and electrode position and contact quality (Adler et al. 1996). Additionally, difference EIT linearises the image reconstruction process, which permits one-step, linear imaging (starting from Sheffield Backprojection (Barber \& Seagar 1987) to the algorithms based on pseudo-inverses of sensitivity matrices, such as NOSER (Cheney et al. 1990), and GREIT (Adler et al. 2009)). Reconstruction algorithms in the latter tradition have used finite element meshes (FEM) to model the body of interest and to calculate a sensitivity (or Jacobian) matrix. The advantage of the FEM is that it facilitates considering arbitrary body shapes and tissue properties.

Most linear EIT algorithms have assumed that image changes occur with respect to an homogeneous background. Such an approach has the advantage of simplicity; however, such an assumption, especially for chest EIT, is clearly unwarranted. The lungs are at least four times more resistive than other thoracic tissue (Gabriel et al. 2009). This means that sensitivity matrices calculated with an homogeneous assumption will dramatically underestimate the EIT sensitivity in the lung region, resulting in inaccuracies in the shape and amplitude of reconstructed images (Grychtol \& Adler 2013).

In this paper, we are motivated to consider a further issue concerning linear EIT algorithms: what tissue property should they reconstruct?

Image reconstruction algorithms have been formulated to consider three tissue properties: 1) conductivity $(\sigma), 2)$ resistivity $(\rho=1 / \sigma)$, and 3) log conductivity $(\log (\sigma)=-\log (\rho))$. As we show below, sensitivity matrices calculated using an 
homogeneous FEM will be identical (to within a scale factor) for the various tissue properties. Thus given this assumption, there has been no motivation to carefully consider the choice of tissue properties.

However, if the FEM background conductivity is not homogeneous, then the calculated sensitivity matrix (on which linear reconstruction is based) will vary depending on the tissue property model chosen. This means that time difference EIT images will differ depending on the reconstructed tissue properties. Interestingly, early work on EIT (then APT) was to reconstruct resistivity (Barber \& Seagar 1987), while more recent work, including algorithms based on EIDORS and GREIT (Adler et al. 2009), formulate reconstruction in terms of conductivity. The transition went largely unnoticed, most likely because images look the same when an homogeneous background is assumed. For lung imaging, EIT image amplitude has generally been considered proportional to lung regional air volume changes, but it has not been common to directly reconstruct lung volumes. In our analysis, we can consider air volumes to be a further parameterization of tissue properties, which can then be analysed by the approach we develop.

This variation in the EIT images produced raises two questions: 1) what magnitude and type of change is produced when different tissue properties are modelled? and 2) what is the "best" choice for these properties? In our paper, we primarily consider the first question, in order to understand the circumstances in which tissue property choice is a key factor to be considered in the interpretation of images. However, we do not feel able to recommend a "best" choice yet. Instead, we propose a program of research which will help clarify the choice.

\section{Methods}

Our goal is to understand the magnitude and types of changes to reconstructed EIT images as the selected tissue property is changed. First, we re-derive the classic equations governing linear image reconstruction (after Adler et al. 2009) in order to clarify the role played by the tissue properties. Next, we use a relatively simple FEM geometry in order to simulate the types of images associated with a lung recruitment manoeuvre (e.g. Frerichs et al. 2003), as a proposed EIT use case. In this model, we calculate the sensitivity matrices and reconstructed images in order to evaluate the effect of the considered tissue properties. Next, using data from a classic EIT animal experiment (Frerichs et al. 2003), we repeat the analyses for reconstructions based on various tissue properties, and consider how this changes the interpretation. Finally, we consider the question of how to establish which of these options is "best".

\subsection{Parameter choice in linear image reconstruction}

Given a specific stimulation current and measurement strategy, the relationship between

potential measurements $\mathbf{v}$ on the domain's surface and the distribution of conductivity 
$\boldsymbol{\sigma}$ within it is a non-linear function $\mathbf{v}=F_{\sigma}(\boldsymbol{\sigma})$ and can be expressed by the Taylor series:

$$
F_{\sigma}(\boldsymbol{\sigma})=F_{\sigma}\left(\boldsymbol{\sigma}_{0}\right)+\left.\sum_{n=1}^{\infty} \frac{1}{n !} \frac{\partial^{n} F_{\sigma}(\boldsymbol{\sigma})}{\partial \boldsymbol{\sigma}^{n}}\right|_{\boldsymbol{\sigma}_{0}}\left(\boldsymbol{\sigma}-\boldsymbol{\sigma}_{0}\right)^{n}
$$

where $\boldsymbol{\sigma}_{0}$ is an arbitrary reference conductivity distribution. Thus, approximating to first order, the difference between two measurements can be expressed as the linear relationship:

$$
\mathbf{d}=F_{\sigma}(\boldsymbol{\sigma})-\left.F_{\sigma}\left(\boldsymbol{\sigma}_{0}\right) \approx \frac{\partial F_{\sigma}(\boldsymbol{\sigma})}{\partial \boldsymbol{\sigma}}\right|_{\boldsymbol{\sigma}_{0}}\left(\boldsymbol{\sigma}-\boldsymbol{\sigma}_{0}\right)=\mathbf{J}_{\sigma} \Delta \boldsymbol{\sigma}
$$

where $\mathbf{J}_{\sigma}$ is the Jacobian, or sensitivity, matrix defined as

$$
\left[\mathbf{J}_{\sigma}\right]_{i, j}=\left.\frac{\partial\left[F_{\sigma}(\boldsymbol{\sigma})\right]_{i}}{\partial[\boldsymbol{\sigma}]_{j}}\right|_{\boldsymbol{\sigma}_{0}}
$$

for each measurement $i$ and element of the domain $j$.

Analogously, the difference data $\mathbf{d}$ can be expressed as a function of the change in resistivity distribution $\boldsymbol{\rho}$ :

$$
\left.\mathbf{d} \approx \frac{\partial F_{\rho}(\boldsymbol{\rho})}{\partial \boldsymbol{\rho}}\right|_{\boldsymbol{\rho}_{0}}\left(\boldsymbol{\rho}-\boldsymbol{\rho}_{0}\right)=\mathbf{J}_{\rho} \Delta \boldsymbol{\rho}
$$

where the Jacobian $\mathbf{J}_{\rho}$ is defined by

$$
\left[\mathbf{J}_{\rho}\right]_{i, j}=\left.\frac{\partial\left[F_{\rho}(\boldsymbol{\rho})\right]_{i}}{\partial[\boldsymbol{\rho}]_{j}}\right|_{\boldsymbol{\rho}_{0}} .
$$

If $\boldsymbol{\rho}_{0}$ and $\boldsymbol{\sigma}_{0}$ are equivalent, i.e. pertain to the same underlying physical reality $\left(\left[\boldsymbol{\rho}_{0}\right]_{i}=\left[\boldsymbol{\sigma}_{0}\right]_{i}^{-1}\right)$, the elements of $\mathbf{J}_{\rho}$ and $\mathbf{J}_{\sigma}$ are related by the chain rule

$$
\begin{aligned}
{\left[\mathbf{J}_{\rho}\right]_{i, j} } & =\left.\frac{\partial\left[F_{\sigma}(\boldsymbol{\sigma})\right]_{i}}{\partial[\boldsymbol{\sigma}]_{j}} \frac{\partial[\boldsymbol{\sigma}]_{j}}{\partial[\boldsymbol{\rho}]_{j}}\right|_{\boldsymbol{\rho}_{0}}=\left[\mathbf{J}_{\sigma}\right]_{i, j} \frac{\partial}{\partial[\boldsymbol{\rho}]_{j}}\left[\boldsymbol{\rho}_{0}\right]_{j}^{-1} \\
& =\left[\mathbf{J}_{\sigma}\right]_{i, j}\left(-\left[\boldsymbol{\rho}_{0}\right]_{j}^{-2}\right)=-\left[\mathbf{J}_{\sigma}\right]_{i, j}\left[\boldsymbol{\sigma}_{0}\right]_{j}^{2}
\end{aligned}
$$

Another common parametrization of tissue properties is the log conductivity $\left(l_{B} \triangleq \log _{B} \sigma=-\log _{B} \rho\right)$, where logarithmic bases, $B$, of $e$ or 10 are common (Brown et al. 1985, Shaw et al. 1993, Lesparre et al. 2013). Using, $\mathbf{l}_{B}$, to represent the distribution of $\log$ tissue properties, $l_{B}, \sigma=B^{l_{B}}=e^{\log (B) l_{B}}$, and

$$
\left.\mathbf{d} \approx \frac{\partial F_{l}(\mathbf{l})}{\partial \mathbf{l}}\right|_{\mathbf{l}_{0}}\left(\mathbf{l}-\mathbf{l}_{0}\right)=\mathbf{J}_{l} \Delta \mathbf{l}
$$

where the Jacobian of $\log$ conductivity, $\mathbf{J}_{l}$ is

$$
\begin{aligned}
{\left[\mathbf{J}_{l}\right]_{i, j} } & =\left.\frac{\partial\left[F_{l}(\mathbf{l})\right]_{i}}{\partial[\mathbf{l}]_{j}}\right|_{\mathbf{l}_{0}} \\
& =\left.\frac{\partial\left[F_{\sigma}(\boldsymbol{\sigma})\right]_{i}}{\partial[\boldsymbol{\sigma}]_{j}} \frac{\partial[\boldsymbol{\sigma}]_{j}}{\partial[\mathbf{l}]_{j}}\right|_{\mathbf{l}_{0}}=\left[\mathbf{J}_{\sigma}\right]_{i, j} \frac{\partial}{\partial[\mathbf{l}]_{j}} e^{\log (B)\left[\mathbf{l}_{B}\right]_{j}} \\
& =\log (B)\left[\mathbf{J}_{\sigma}\right]_{i, j} e^{\log (B)\left[\mathbf{l}_{B}\right]_{j}}=\log (B)\left[\mathbf{J}_{\sigma}\right]_{i, j}\left[\boldsymbol{\sigma}_{0}\right]_{j}
\end{aligned}
$$


The Jacobian matrices for each tissue property may be related by a diagonal scaling matrix, $\mathbf{S}$, such that $\mathbf{J}_{\rho}=\mathbf{J}_{\sigma} \mathbf{S}_{\rho}$, and $\mathbf{J}_{l_{K}}=\mathbf{J}_{\sigma} \mathbf{S}_{l_{k}}$, where

$$
\begin{aligned}
& {\left[\mathbf{S}_{\rho}\right]_{i, i}=-[\boldsymbol{\sigma}]_{i}^{2},} \\
& {\left[\mathbf{S}_{l_{B}}\right]_{i, i}=\log (B)[\boldsymbol{\sigma}]_{i}}
\end{aligned}
$$

Linear time-difference reconstruction algorithms calculate an estimate $\hat{\mathbf{m}}$ for the image of change in electrical properties $\mathbf{m}($ e.g. $\mathbf{m}=\Delta \boldsymbol{\sigma})$ as

$$
\hat{\mathbf{m}}=\mathbf{R d}
$$

where the reconstruction matrix $\mathbf{R}$ represents a pseudo-inverse of the sensitivity matrix $\left(\mathbf{R}=\mathbf{J}^{\dagger}\right)$. In most cases, $\mathbf{R}$ can be defined by the solution which minimizes a norm composed of data mismatch and image penalty functions:

$$
\hat{\mathbf{m}}=\underset{\mathbf{m}}{\operatorname{argmin}}\|\mathbf{W}(\mathbf{d}-\mathbf{J} \mathbf{m})\|^{2}+\alpha^{2}\|\mathbf{L m}\|^{2}
$$

in which matrices $\mathbf{W}$ and $\mathbf{L}$ represent a priori models of data and image distributions. $\mathbf{W}$ models the reliability of each measurement channel and $\mathbf{L}$ models the expected amplitude and spatial correlations of image elements. The hyperparameter $\alpha$ controls the compromise between fidelity to the data and to image constraints. As $\alpha$ increases, image resolution decreases, but reconstruction robustness to noise improves. Based on 12 , the linear reconstruction matrix, $\mathbf{R}$ is calculated:

$$
\mathbf{R}=\left(\mathbf{J}^{t} \mathbf{W}^{t} \mathbf{W} \mathbf{J}+\alpha^{2} \mathbf{L}^{t} \mathbf{L}\right)^{-1} \mathbf{J}^{t} \mathbf{W}^{t} \mathbf{W}
$$

We use the common assumption that $\mathbf{W}=\mathbf{I}$ (i.e. all measurement channels have identical noise amplitude). Two models for $\mathbf{L}$ are used: 1) the Laplace prior (Polydorides \& Lionheart 2002), which penalizes non-smooth images, and 2) the NOSER prior (Cheney et al. 1990), which penalizes image amplitudes in proportion to their sensitivity in the measured data.

Thus, the linear reconstruction matrix $\left(\mathbf{R}=\mathbf{J}^{\dagger}\right)$ is a function of the sensitivity matrix; we use the notation $\mathbf{J}_{*}^{\dagger}$ to indicate the value calculated using a particular $\mathbf{J}_{*}$, calculated from particular tissue properties, *. Analogously, we refer to the images reconstructed with $\mathbf{J}_{*}^{\dagger}$ as $\hat{\mathbf{m}}_{*}=\mathbf{J}_{*}^{\dagger} \mathbf{d}$. It is clear from equations 3 and 6 that if the reference conductivity $\boldsymbol{\sigma}_{0}$ is homogeneous, $\hat{\mathbf{m}}_{\sigma}, \hat{\mathbf{m}}_{\log }$ and $\hat{\mathbf{m}}_{\rho}$ differ only by a scalar factor and so appear identical if scaled appropriately to use the same colour map.

\subsection{Amplitude response map}

In order to describe the distribution of reconstructed sensitivity across the image, we use the "amplitude response map" (Grychtol et al. 2012), ARM, defined such that each image pixel, $i$, represents the total image amplitude produced by a small change in a spherical target centred at that pixel's position. Specifically, given a small change in tissue properties $\Delta \mathbf{m}_{i}$, which is zero everywhere except for elements which are contained in or intersect the spherical target $i$, the measured data, $\mathbf{d}$, and reconstructed model $\hat{\mathbf{m}}$ 
are

$$
\begin{aligned}
\mathbf{d}_{i} & =\mathbf{J}_{p} \mathbf{m}_{i} \\
\hat{\mathbf{m}}_{i} & =\mathbf{R}_{r} \mathbf{d}_{i}=\mathbf{R}_{r} \mathbf{J}_{p} \mathbf{m}_{i}
\end{aligned}
$$

in which $p$ is the assumed tissue properties for the forward model; data, $\mathbf{d}_{i}$ are calculated from the sensitivity of the forward model to tissue properties $p$, because $\mathbf{m}_{i}$ is a small change in the units of $p$. Here, $r$ is the assumed tissue properties for the reconstruction. In this case, the amplitude response in $i$ is the sum (weighted by image element area) of the reconstructed model, $\mathrm{ARM}_{i}=\mathbf{a}^{t} \hat{\mathbf{m}}_{i}$, where $\mathbf{a}$ is a vector of element areas (which is uniform if reconstructing onto a pixel array). Based on 14 , we create a matrix $\mathbf{T}$ which corresponds to the targets for each pixel, such that each column, $\operatorname{col}_{i} \mathbf{T}=\mathbf{m}_{i}$.

$$
\mathrm{ARM}=\mathbf{a}^{t} \mathbf{R}_{r} \mathbf{J}_{p} \mathbf{T}
$$

It is worth commenting on the use of two different tissue properties - for forward modelling, $p$, and reconstruction, $r$ - in our analysis. As we note in section 2.3, the relationship between lung volume and the change in tissue electrical properties is not exactly known. However, in this paper we seek to understand the effect of the assumed tissue properties on the reconstructed images. Thus, for a fair comparison between reconstructions $(r)$, a common set of "real underlying" tissue properties, $p$, must be assumed.

\subsection{Lung resistivity and air volume}

Lung tissue is electrically resistive because the contained air spaces force electrical current (especially at the relatively low frequencies used in EIT) to flow largely through the parenchymal tissue and fluids. Over the physiological range, it has typically been assumed that EIT images have an amplitude which is linearly proportional to lung volume, as has been shown in several EIT studies (e.g. Adler et al. 1997). Most of these studies are limited by the fact that a relatively small range of lung volumes (typically between functional residual capacity, FRC, and total lung capacity, TLC) are studied. Lung electrical properties have also been studied in ex-vivo conditions (see e.g. Gabriel, Gabriel \& Corthout (1996), Gabriel, Lau \& Gabriel (1996) and references therein). However, measurements have typically been made only at a limited number of points on the inflation curve. Additionally, methodological issues make placement of electrodes and electrical measurements on the surface of the lung difficult.

As mentioned previously, for this paper, it is only essential that the lung tissue model be reasonable, and be used consistently across the different reconstruction algorithms. We assume that lung resistivity is proportional to volume. This is consistent with the model of Nopp et al. (1997) and may be justified as follows: if electrical current flows only through lung fluid, blood and parenchymal regions, then the lung will behave like a resistor network. Further, if conductive material in the lungs is incompressible and electrically isotropic, then the resistance, $R$, of each element in the network will increase with strain, $\epsilon$, by lengthening and reduction in cross sectional area, thus, $R \propto(1+\epsilon)^{3}$. 
Since lung volume is also proportional to $(1+\epsilon)^{3}$, a homogeneously expanding lung will have resistance proportional to volume.

We choose the coefficients of the linear relationship between resistivity and volume based on the lung conductivity values at $100 \mathrm{kHz}$ reported by Gabriel et al. (2009): 0.08-0.27 S/m for deflated and $0.03-0.09 \mathrm{~S} / \mathrm{m}$ for inflated lung. The resistivity values corresponding to the extremes of the observed range are 3.7 and $33.3 \Omega \mathrm{m}$ for deflated and inflated lung, respectively. We assume those values correspond to lung volumes of $1.2 \mathrm{~L}$ (average residual lung volume in men) and 6.0 L (total lung volume). This yields the function $f_{\rho}(V)$ relating resistivity $\rho$ in $\Omega \mathrm{m}$ and lung volume $\mathrm{V}$ in $\mathrm{L}$ :

$$
f_{\rho}(V)=6.1728 V-3.7037 \text {. }
$$

Consequently, we obtain

$$
\begin{aligned}
& f_{\sigma}(V)=\frac{1}{f_{\rho}(V)} \\
& f_{\log }(V)=\log \left(f_{\rho}(V)\right) .
\end{aligned}
$$

\subsection{Recovering lung volume changes}

The physical units of a difference EIT reconstruction depend on the choice of reconstructed tissue properties. Thus, the faithfulness to reality of images obtained with different parametrizations cannot readily be compared. To facilitate such comparison, they can, in theory, all be converted to the same units, by working backward from the assumptions. Difference EIT reconstructs the change in distribution of electrical properties within a domain with respect to some (assumed) reference distribution. Thus, by adding the reference distribution to the reconstructed image, one should obtain an image representing the current distribution in absolute values, which could then easily be converted to different physical units or, given appropriate model, to lung volume. Unfortunately, because of the shortcomings of difference EIT images such as overshoot or ringing and potential inaccuracies in the assumed reference distribution, this approach rarely works in practice, producing for example negative conductivity values.

We therefore use a different approach. If the change in lung volume $\Delta V=V_{1}-V_{0}$ is sufficiently small, we can assume (analogously to eq. 1) that

$$
\Delta p=\left.\frac{d f_{p}(V)}{d V}\right|_{V_{0}} \Delta V=K_{p} \Delta V
$$

for some electrical property $p$, and a reference volume $V_{0}$ around which we linearize, where $f_{p}$ is one of the functions defined in Eq. 16 18. Thus, using the assumed electrical property background $\mathbf{p}_{0}$, the corresponding volume distribution $\mathbf{V}_{0}=f_{\mathrm{p}}^{-1}\left(\mathbf{p}_{0}\right)$ and the associated vector $\mathbf{K}_{p}$ as defined in Eq. 19 above, we can attempt to recover an image of volume changes $\mathbf{m}_{\mathrm{vol}}$ from a difference EIT image $\mathbf{m}_{p}$ as

$$
\hat{\mathbf{m}}_{\mathrm{vol}}=\operatorname{diag}\left(\mathbf{K}_{p}\right)^{-1} \hat{\mathbf{m}}_{p} \text {. }
$$


The conversion factor can also be incorporated into the reconstruction matrix, such that

$$
\hat{\mathbf{m}}_{\mathrm{vol}}=\operatorname{diag}\left(\mathbf{K}_{p}\right)^{-1} \mathbf{R}_{p} \mathbf{d}=\mathbf{R}_{p, \mathrm{vol}} \mathbf{d} .
$$

The value of each element of the image $\hat{\mathbf{m}}_{\mathrm{vol}}$ can then be interpreted as the total lung volume change that would result from the entire lung undergoing the same density change as that element. Thus, although expressed in volume units, $\hat{\mathbf{m}}_{\mathrm{vol}}$ is best thought of as showing density changes. If the volume represented by each image element is known, it could be further converted into local air volume changes.

It is worth noting that when $\mathbf{V}_{0}$ is homogeneous or the derivative of $f_{p}$ is independent of $V_{0}$ (as is the case for $f_{\rho}$ above), all elements of $\mathbf{K}_{p}$ are equal and so the conversion to volume units has no effect on the appearance of the reconstructed images.

\subsection{Simulated measurements}

Using EIDORS 3.7讯and Netgen 5.0 (Schöberl 1997), we simulate voltage measurements on a cylindrical tank with 16 electrodes and two resistive objects representing the lungs (figure 1a). We use the Sheffield stimulation and measurement protocol (Brown \& Seagar 1987).

The lungs are divided into "upper" and "lower" halves with different conductivities.

By manipulating the electrical properties of the lungs according to eq. 16, we simulate non-homogeneously distributed changes in air volume (tidal volume, TV). The electrical properties elsewhere in the simulated tank are kept constant at $0.48 \mathrm{~S} / \mathrm{m}(2.083 \Omega \mathrm{m})$. We assume the upper lung to be more resistive to represent a decrease in end-expiratory lung volume (EELV) along the gravitational axis (Pelosi et al. 1994). We simulate four scenarios with different EELV and TV. The specific values as well as the corresponding electrical properties are given in table 1. As required for difference EIT, we simulate two measurements for each scenario: one at EELV and another at EELV + TV.

\subsection{Image reconstruction}

To reconstruct images from the simulated data, we use the dual-model approach whereby the sensitivity matrix is calculated on a 3D cylindrical model, but parameters are reconstructed onto a $2 \mathrm{D}$ pixel grid at the plane of electrodes. The mapping between the $3 \mathrm{D}$ and $2 \mathrm{D}$ models assumes that electrical properties do not change along the axis of the cylinder. To avoid the inverse crime (Lionheart 2004), the 3D model used for reconstruction is coarser than that from which simulated measurements are obtained (the forward model contains 163209 and the inverse model 23165 simplex elements).

Simulated difference measurements are reconstructed as changes in conductivity, log conductivity and resistivity. The distribution of electrical properties corresponding to the reference measurement at EELV is either assumed to be homogeneous or corresponds to that used in generating the measurements (true background). We use the one-step

$\ddagger$ http://eidors3d.sf.net 
Table 1: Simulation settings.

\begin{tabular}{cccccccccc}
\hline No. & half & $\begin{array}{c}\text { EELV } \\
{[\mathrm{L}]}\end{array}$ & $\begin{array}{c}\text { TV } \\
{[\mathrm{L}]}\end{array}$ & $\begin{array}{c}\sigma \\
{[\mathrm{S} / \mathrm{m}]}\end{array}$ & $\log \sigma$ & $\begin{array}{c}\rho \\
{[\Omega \mathrm{m}]}\end{array}$ & $\begin{array}{c}\Delta \sigma \\
{[\mathrm{S} / \mathrm{m}]}\end{array}$ & $\Delta \log \sigma$ & $\begin{array}{r}\Delta \rho \\
{[\Omega \mathrm{m}]}\end{array}$ \\
\hline 1 & $\mathrm{u}$ & 1.0 & 0.50 & 0.12 & -2.16 & 8.64 & -0.05 & -0.54 & 6.17 \\
1 & $\mathrm{l}$ & 0.5 & 0.00 & 0.41 & -0.90 & 2.47 & 0.00 & 0.00 & 0.00 \\
2 & $\mathrm{u}$ & 1.0 & 0.40 & 0.12 & -2.16 & 8.64 & -0.04 & -0.45 & 4.94 \\
2 & $\mathrm{l}$ & 0.5 & 0.10 & 0.41 & -0.90 & 2.47 & -0.14 & -0.41 & 1.23 \\
3 & $\mathrm{u}$ & 1.0 & 0.25 & 0.12 & -2.16 & 8.64 & -0.03 & -0.31 & 3.09 \\
3 & $\mathrm{l}$ & 0.5 & 0.25 & 0.41 & -0.90 & 2.47 & -0.23 & -0.81 & 3.09 \\
4 & $\mathrm{u}$ & 1.0 & 0.25 & 0.12 & -2.16 & 8.64 & -0.03 & -0.31 & 3.09 \\
4 & $\mathrm{l}$ & 1.0 & 0.25 & 0.12 & -2.16 & 8.64 & -0.03 & -0.31 & 3.09 \\
\hline \multicolumn{7}{c}{ half - upper (u) } & or lower (l) part of the simulated lungs; EELV - end- \\
& expiratory lung volume; TV - tidal volume; $\sigma-$ conductivity; $\rho-$ resistivity.
\end{tabular}

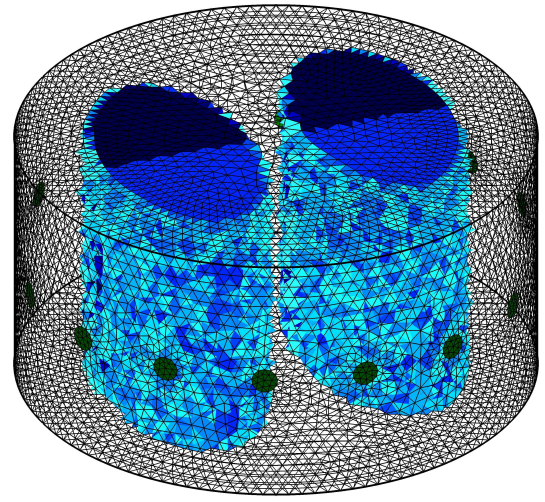

(a)

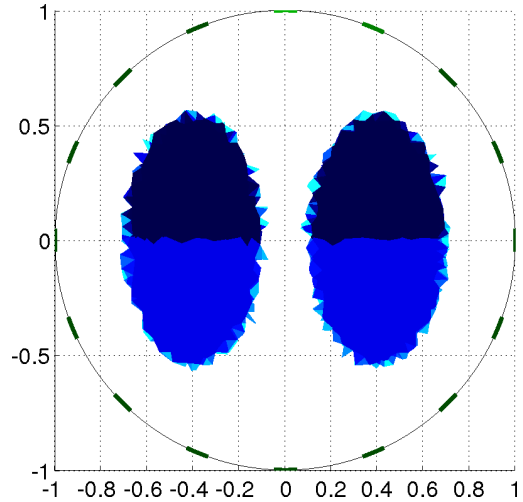

(b)

Figure 1: FEM setup for lung background conductivity investigation: (a) 3D view; (b) 2D cut through at the level of the electrodes. Colour code: navy — ventral lung (less conductive); blue - dorsal lung (more conductive). Electrodes are indicated in green.

Gauss-Newton reconstruction algorithm (eq. 13) with the NOSER (Cheney et al. 1990) prior, keeping the hyperparameter constant for all reconstructions $(\alpha=0.03)$.

\section{Results}

The sensitivity of measurements to changes in tissue properties in the plane of electrodes expressed as conductivity, log conductivity and resistivity for the same scenario are depicted in figure 2, aggregated as absolute values for all 16 current stimulations. In agreement with the inverse relation between conductivity and resistivity, unit increase in conductivity causes bigger changes in measurements when happening in less conductive lung areas, while the opposite is true for unit increase in resistivity. For all parametrizations, sensitivity decreases toward the centre of the medium.

The simulation parameters for the four scenarios are presented in figure 3 (top two rows) together with the corresponding reconstructions using homogeneous 


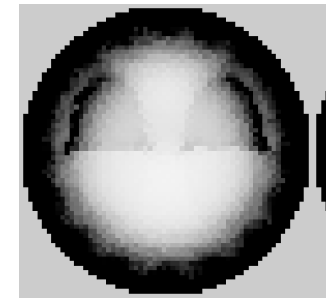

(a)

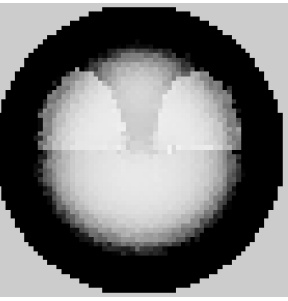

(b)

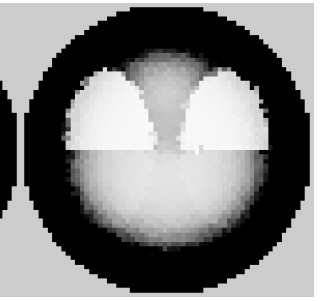

(c)

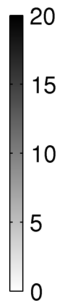

\section{0}

Figure 2: Sensitivity of the measurements to unit changes in a) conductivity, b) $\log$ conductivity, and c) resistivity in the plane of electrodes with simulated air volumes of 1 $\mathrm{L}$ in the upper lung and 0.5 in the lower lung. The colour scale is adjusted to show the effects in the lungs, details near the boundary are lost.

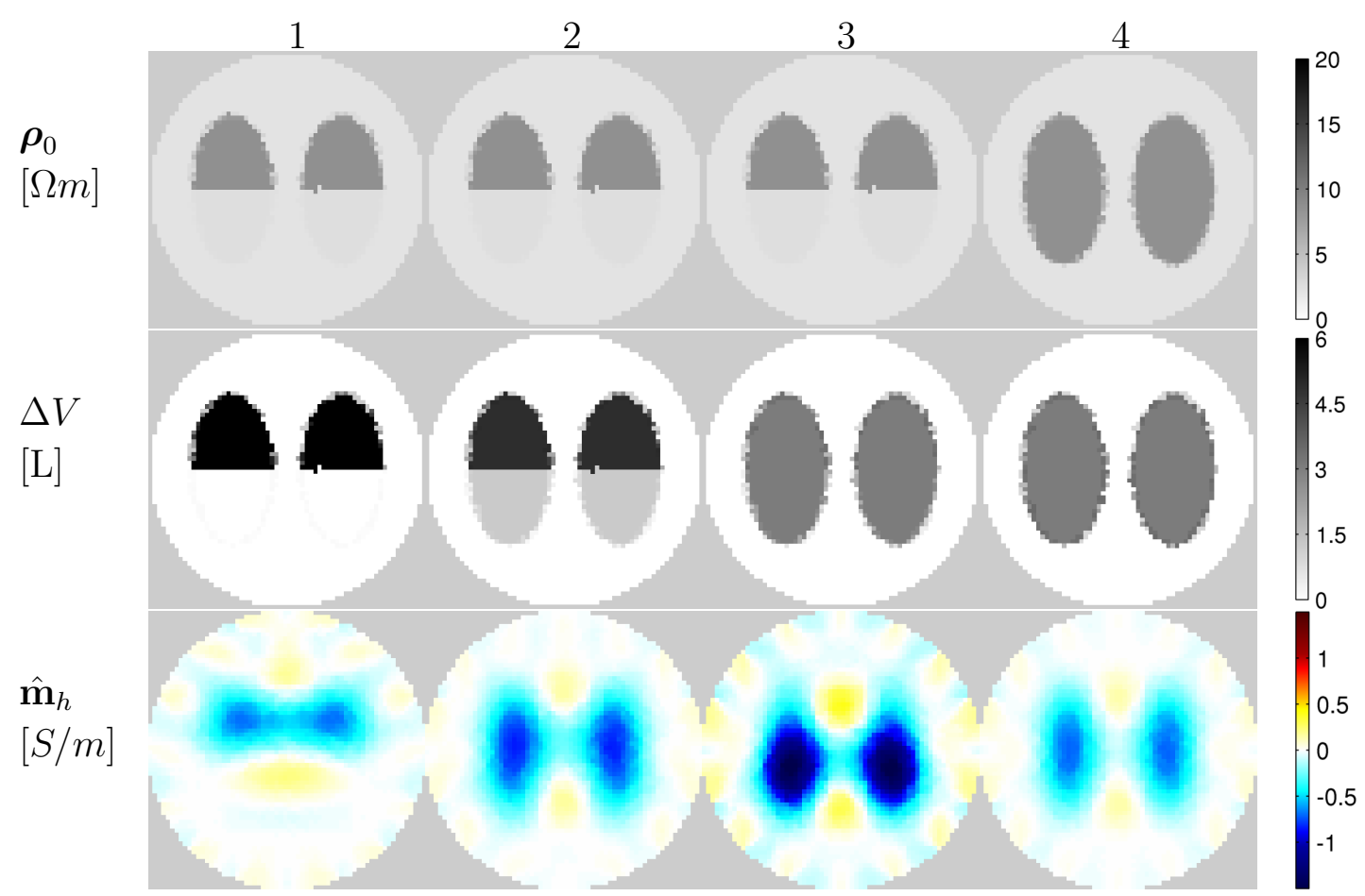

Figure 3: Simulated background resistivity distribution $\boldsymbol{\rho}_{0}$ (top) and tidal volume change between difference EIT frames $\Delta V$ (middle), and the corresponding EIT images for different cases of lung recruitment (columns, cf. Table 1) reconstructed as conductivity change $\hat{\mathbf{m}}_{h}$ assuming homogeneous background (bottom).

background $\left(\hat{\mathbf{m}}_{h}\right)$. The reconstructions do not correspond well to the simulated volume changes; changes in the lower, less resistive part of the lung are overestimated.

In the top panel of figure 4 reconstructions obtained when using the correct linearisation point (true background) are shown. The effect observed for reconstructions with homogeneous background is even more dramatic when data are reconstructed as conductivity change $\left(\hat{\mathbf{m}}_{\sigma}\right)$ using the true background. Reconstructions with true background with changes parametrized as $\log$ conductivity $\left(\hat{\mathbf{m}}_{\log }\right)$ are comparable with those obtained with homogeneous background. Predictably (since we assumed resistivity is proportional to air volume), reconstructions of resistivity changes $\left(\hat{\mathbf{m}}_{\rho}\right)$ using the true 


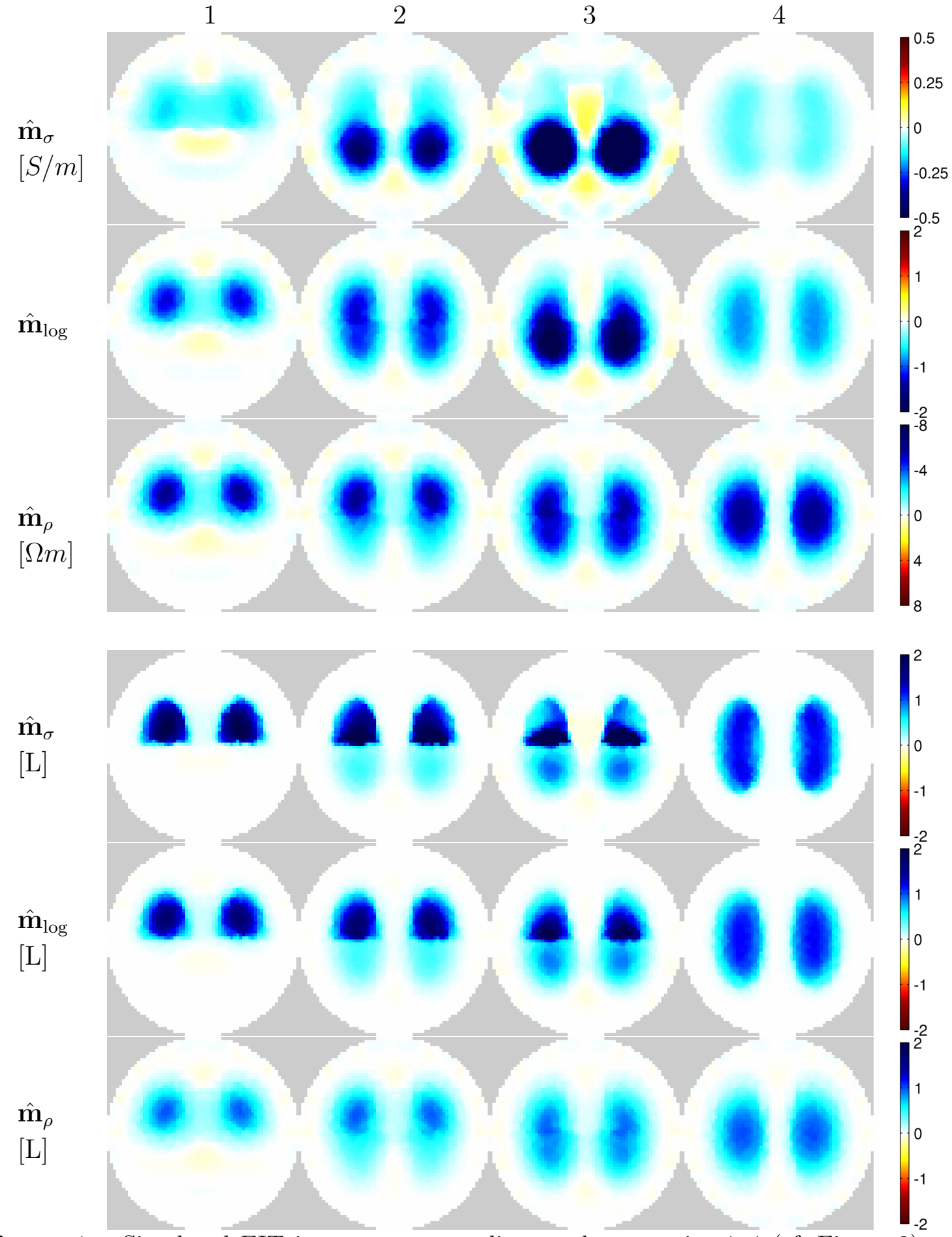

Figure 4: Simulated EIT images corresponding to the scenarios 1-4 (cf. Figure 3) using the true background as the linearisation point and reconstructed as conductivity $\left(\hat{\mathbf{m}}_{\sigma}\right), \log$ conductivity $\left(\hat{\mathbf{m}}_{\mathrm{log}}\right)$, and resistivity $\left(\hat{\mathbf{m}}_{\rho}\right)$ changes. Top panel: images in original units; bottom panel: converted to volume changes as outlined in section 2.4. Note that the colour scales for resistivity and volume changes are inverted to facilitate comparison. 


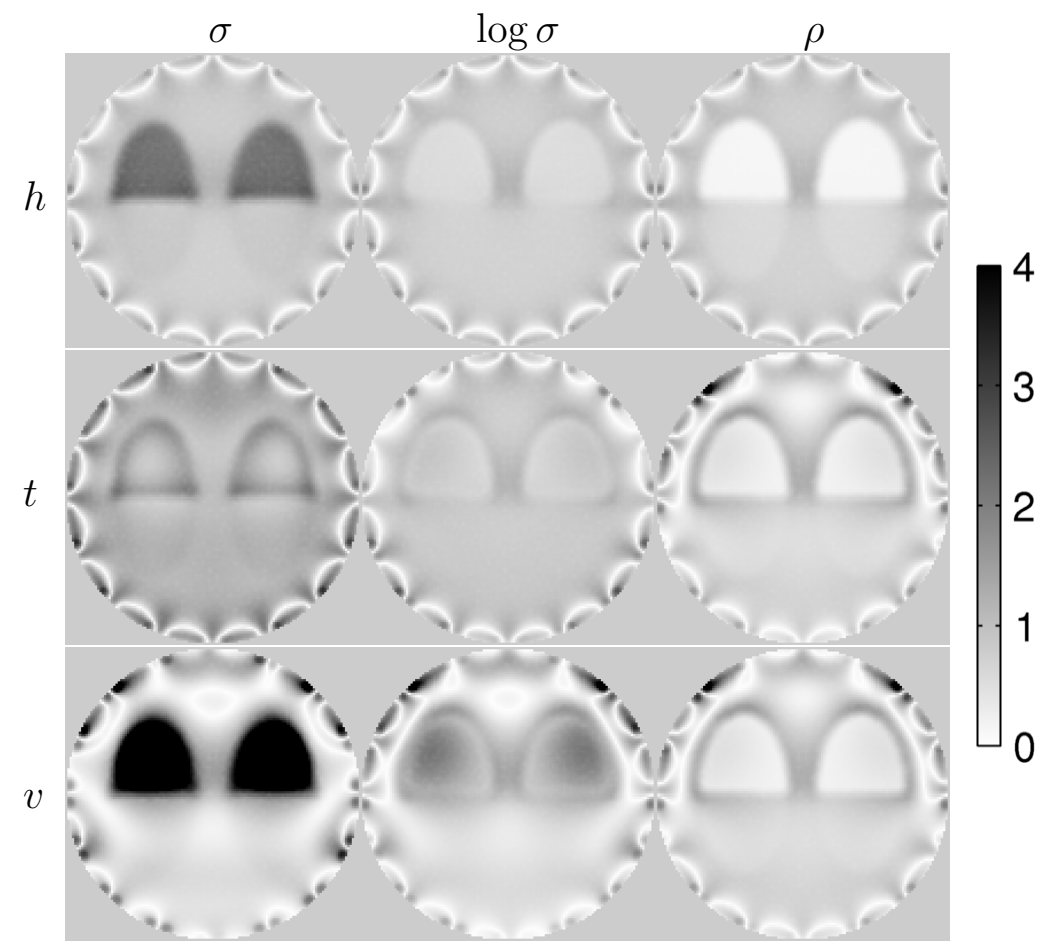

Figure 5: Amplitude response maps (ARM) corresponding to simulations 1-3 with nonhomogeneous lungs (cf. figure 2) when reconstructing (left to right) conductivity $(\sigma), \log$ conductivity $(\log \sigma)$ and resistivity $(\rho)$. $h$ : reconstructions using an homogeneous background; $t$ : reconstructions using the true background; and $v$ : reconstructions using the true background converted to volume units as described in section 2.4. Darker shading reflects higher amplitude response; images for $h$ and $t$ are scaled such that the central region is the same shade.

background best reflect the distribution of tidal volume changes, but not their amplitude. The maximum image amplitude should be higher when the volume change is confined to half the lung (scenarios 1 and 2).

The result of applying equation 20 to the reconstructed images in order to recover lung volume changes (volume images) is presented in the lower panel of figure 4 . On the whole, volume images obtained from conductivity and log conductivity parametrizations reflect more closely the simulated tidal volume changes (middle row in figure 33). They also show the effective cropping of the original reconstructions (top panel) to the assumed lung shape, where image values are selectively amplified, thus reducing artefacts outside the lung region. However, clear discontinuities are introduced and the distribution of tidal volume shows unexpected inhomogeneities. This is particularly visible for images corresponding to scenario 3 where homogeneous tidal volume was simulated (cf. figure 3). In contrast, for resistivity reconstructions conversion to volume results in uniform scaling and so the appearance of the images is not altered.

Amplitude response maps for reconstructions with true and homogeneous background, and as volume changes for the three parametrisations are presented in figure 5. The maps show significant inhomogeneities, especially in the image area corresponding to the more resistive upper lung. Changes in that area are 
overestimated when reconstructed as conductivity changes, and underestimated in resistivity reconstruction. Log conductivity shows least inhomogeneities. For all parametrisations, ARMs show less contrast in the upper lung when the true background is used for reconstructions. In comparision, ARMs obtained from volume change reconstructions using the conductivity parametrization show a strong amplifying effect for changes in the upper lung, and suppression elsewhere. This effect is also present but weaker for the log conductivity parametrization, but absent for resistivity parametrization, since conversion to volume does not affect the distribution of image values. The white arches between adjacent electrodes represent a transition between negative and positive sensitivity.

\section{Discussion}

In this paper, we have investigated the question of the choice of tissue properties on which to base the calculation of the linear EIT reconstruction matrix. Specifically, our goal was to investigate and understand the type and magnitude of the differences between reconstructions of the same data as changes in conductivity $\left(\hat{\mathbf{m}}_{\sigma}\right)$, resististivity $\left(\hat{\mathbf{m}}_{\rho}\right)$, and $\log$-conductivity $\left(\hat{\mathbf{m}}_{\log }\right)$. We focus on sensitivity-based linear reconstruction algorithms for difference EIT.

First, we describe the derivation of the EIT sensitivity (Jacobian) matrix, $\mathbf{J}$, and its explicit dependence on an assumed background around which time-difference changes occur. Since the FEM is most naturally written in terms of the tissue conductivity, $\mathbf{J}$ for other tissue properties appears as $\mathbf{J}_{\sigma} \mathbf{S}$, where $\mathbf{S}$ is a diagonal matrix, whose individual values depend on the assumed conductivity background and the chosen tissue property model. When an assumption of an homogeneous background (linearisation point) is made, $\mathbf{S}$ is a scaled identity matrix, and does not change the spatial distribution of images. However, when the background contains contrast, such as the lungs (Grychtol \& Adler 2013), S non-uniformly scales the sensitivity matrix. This affects the spatial distribution of images obtained with linear sensitivity-based reconstruction algorithms.

Next, using a 3D cylindrical FEM model with elliptical lung regions, we explore the effect of three properties. In figure 3 and figure 4, lungs at four different stages of recruitment are simulated. A geometrically simple model was chosen in order to ensure the effects were due to the tissue properties under investigation, and not the FEM geometry. The spatial distribution of reconstructed images varies dramatically: compared to the homogeneous case $\hat{\mathbf{m}}_{h}$, reconstructions $\hat{\mathbf{m}}_{\sigma}$ and $\hat{\mathbf{m}}_{\log }$ have larger image amplitude in dependent (lower) lung regions, while $\hat{\mathbf{m}}_{\rho}$ has more non-dependent amplitude, and most accurately reflects the simulated changes in lung volume (which we assumed are linearly related to changes in resistivity). None of the studied parametrizations correctly reconstruct both the distribution and the amplitude of the simulated tidal volume change.

Attempts to recover volume changes from the reconstructed changes in electrical properties result in images that more closely resemble the simulated distribution of tidal 
$[\mathrm{OU}]$
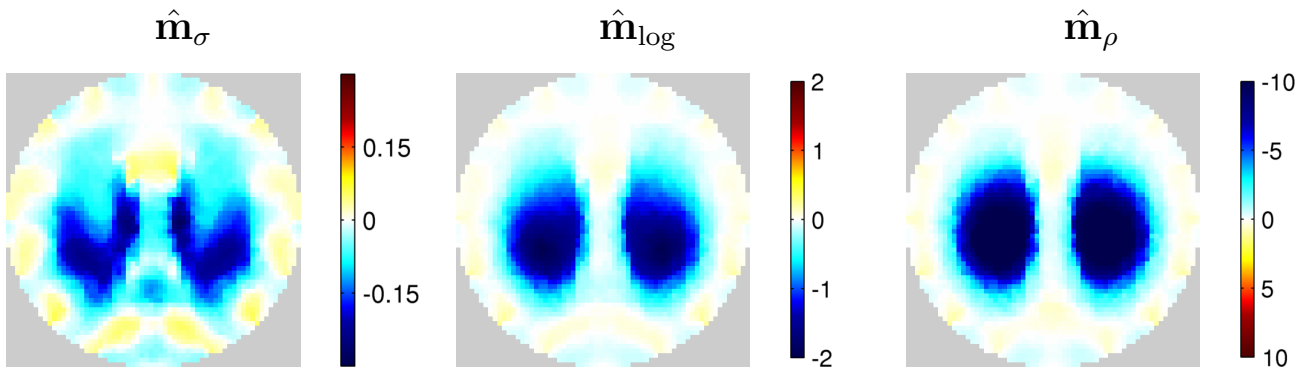

$[\mathrm{L}]$
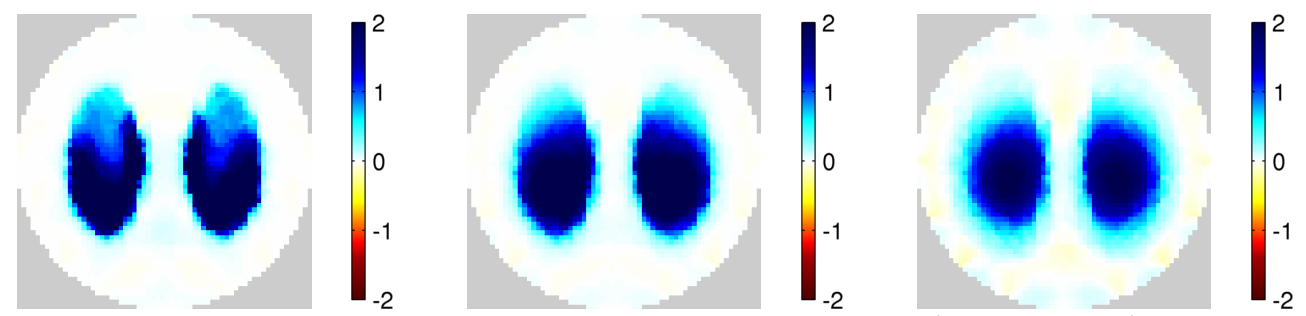

Figure 6: Simulated EIT images corresponding to scenario 3 (cf. Figure 3 ) reconstructed as conductivity $\left(\hat{\mathbf{m}}_{\sigma}\right), \log$ conductivity $\left(\hat{\mathbf{m}}_{\mathrm{log}}\right)$, and resistivity $\left(\hat{\mathbf{m}}_{\rho}\right)$ changes using the background of scenario 4 (homogeneous, resistive lungs) as linearisation point, presented in original units (OU) and after conversion to volume units (L) as per section 2.4.

volume, thus validating the reconstructions. However, as a result of selective image value amplification and the assumption of linearity between lung volume and electrical properties, pronounced artefacts are introduced by this process.

To further study the effect parametrisation choice has on the distribution of values in the reconstructed image, we use the amplitude response map (ARM), which reflects the forward and inverse model effects. In the ideal world, the contribution from all image regions are identical, and ARM is uniform. Figure 5 shows (top row) how the changes in sensitivity from the tissue model produce contrasts in the lung regions under an homogeneous reconstruction model. This effect is partially compensated (middle row) by background models in the reconstructed images, but "ringing"-type artefacts are introduced near the image boundaries.

Unfortunately, in reality the true background distribution is rarely known, because lung disease is heterogeneous and dynamic. Since the linearisation point used to calculate the reconstruction matrix is therefore necessarily inaccurate, the question arises whether using an homogeneous background is not better than a more realistic but still wrong distribution. We have previously shown that due to the off-centre position of the heart, assuming an homogeneous background results in unduly asymmetric images (Grychtol \& Adler 2013), suggesting it is better to use more realistic backgrounds. One approach is to use a simplified and idealized background distribution, where only the major organs are present (perhaps just the lungs) and conductivity is homogeneous in each. We used this approach to produce the images presented in figure 6 where data from scenario 3 (homogeneous tidal-volume, heterogeneous lungs) are reconstructed using the background of scenario 4 (homogeneous lungs) to calculate the reconstruction matrix. Reconstructions using resistivity parametrization and this "fixed" background 

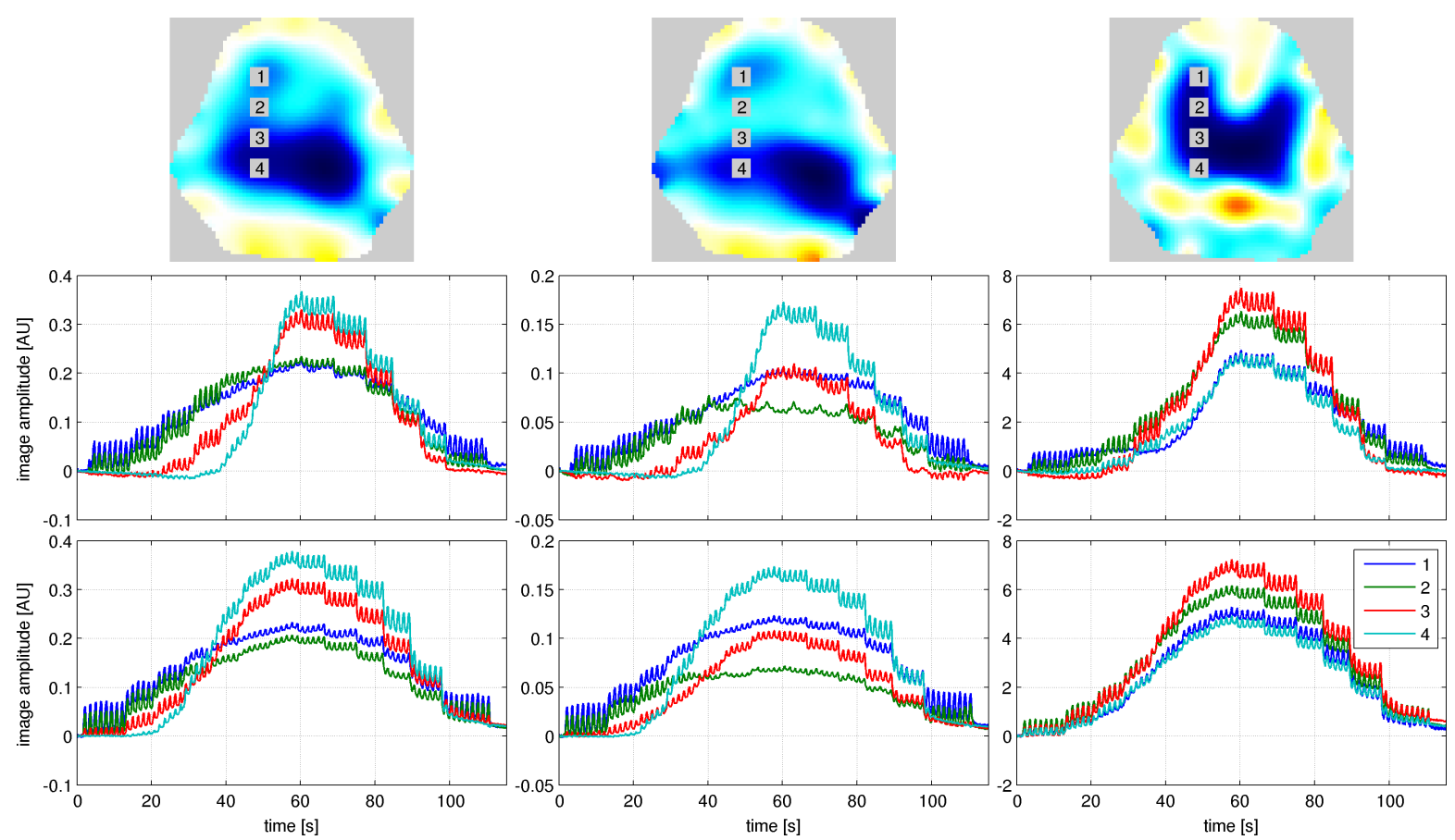

Figure 7: EIT recordings of a stepwise PEEP increase and decrease in a porcine model of lung injury. Reconstructions with a homogeneous model (left), as conductivity change (middle) and resistivity change (right). Top row: image at maximum volume with four regions of interest (ROI) marked; Middle row: tracings of the four ROIs before surfactant treatment, Bottom row: tracings after treatment.

more accurately reflect the true distribution of tidal volume than those using an homogeneous background (figure 3), but less accurately than those using the true background (figure 4). Reconstructions for the other two parametrizations show similar trend showing a distribution in between that obtained with the homogeneous and true backgrounds. However, the images appear less regular and show stronger ringing artefacts.

Since the simulations indicate that the vertical position of reconstructed contrasts are affected, we were interested to see if this effect was also evident in-vivo. A classic experimental data set was chosen (Frerichs et al. 2003), where a piglet had lung injury induced by bronchoalveolar lavage, and experiments were then performed before and after treatment with a surfactant. After placement of thoracic EIT electrodes, EIT scans were taken during ventilation while positive end-expiratory pressure (PEEP) was stepwise incremented and decremented. In figure 7, we reconstruct these data, using the average of the first ten frames as a reference; each column shows reconstructions for different choice of tissue properties. Here, we use the one-step Gauss-Newton algorithm with the Laplace prior (Polydorides \& Lionheart 2002), choosing the value of the hyperparameter such that the Noise Figure (Adler et al. 2009) in the lung region is equal 0.5. We used the standard pig_23kg_16el_lungs mesh as the forward model and assumed lung conductivity equivalent to total lung volume of $2 \mathrm{~L}(0.12 \mathrm{~S} / \mathrm{m})$ and 0.48 $S / m$ for other tissues. Graphs show the time course of pixel intensities (in arbitrary 
units since we use normalized time difference data here) at four indicated locations. The key message is the dramatic changes in the pixel time courses. For example, in the $\hat{\mathbf{m}}_{\sigma}$ reconstruction, pixel $\# 1$ is much larger. The time courses are also affected, the slope of pixels \#1 and \#2 during the incremental phase are larger for $\hat{\mathbf{m}}_{h}$ and $\hat{\mathbf{m}}_{\sigma}$ than for $\hat{\mathbf{m}}_{\rho}$. Converting the images to volume units does not change these observations since we compare small image regions that all fall within the homogeneous lung shape assumed in the forward model. These variations are important, because the distribution of EIT images in the gravitational axis is understood to be a key parameter for clinical interpretation of ventilation distribution (Adler et al. 2012).

We review our motivating questions; first, what magnitude and type of change is produced when different tissue properties are modelled? Based both on simulated and experimental results, the choice of tissue properties for the image reconstruction makes a significant difference. The primary effect appears to be a change in the vertical distribution of image distribution. This may be simplistically characterized as follows: compared to the homogeneous case, $\hat{\mathbf{m}}_{\rho}$ (and to a lesser extent $\hat{\mathbf{m}}_{\log }$ ) "stretch out" changes throughout the lung shape, while $\hat{\mathbf{m}}_{\sigma}$ "pushes" changes further toward less conductive lung regions. This has a dramatic effect on the time course of individual pixels for physiological interpretation of the images.

The next question was: what is the "best" choice for these properties? This study was intended to investigate the effects of tissue properties, and is not comprehensive enough to recommend a best choice; however, it is appropriate to propose a way forward to identify the "best" parameter choices. Both the EIT background model and the tissue parametrization change the reconstruction sensitivity of EIT. We therefore propose two directions for future work.

First, our results suggest that it is advantageous to use the tissue property that is most linearly related to changes in lung volume over the physiological range. Hence, research effort should be directed at developing a detailed model of the relationship between lung volume and its electrical properties in vivo.

Second, the linearization point could be dynamically updated based on either lowresolution absolute EIT, or an analysis of the dynamic EIT signals in tandem with the ventilator settings and a model of lung physiology, enabling the estimation of the physiological state of different lung regions within the EIT images (e.g. Grychtol et al. 2010). Alternatively, it could be derived based on an analysis of the amplitude response. As identified by Adler et al. (2009), the amplitude response of EIT is the most important parameter of image reconstruction for its clinical use. To interpret EIT images, it is important to know if a volume of air has a constant image representation, no matter where in the lungs it is. Thus, we propose that the key metric is the amplitude response map (ARM), which represents the amplitude response of a constant volume change in the image. The best linearization point is the one that optimizes ARM, i.e. which provides constant ARM within a clinically significant region of interest. As shown in figure 5, the homogeneous assumption results in perturbed response in the lung regions; however, the tissue models considered in this paper create a more uniform 
response in the centre of the lung regions at the cost of variations at the lung boundaries. Thus, it appears - in order to optimize the ARM - it will be necessary to model a background conductivity with a smoother transition from tissue to the lung regions. Subsequently, it will be necessary to validate this approach experimentally.

In summary, our results show that the choice of tissue properties is significant for time-difference EIT, when non-homogeneous background models are considered. The effect is to "shift" contrasts in the lung regions. In order to ensure the ability to consistently interpret thoracic EIT images clinically, these results suggest that it is important to perform the algorithmic and experimental work to best compensate for this effect.

\section{Acknowledgements}

BG is grateful for the support provided by the Alexander von Humboldt Research Fellowship.

\section{References}

Adler A, Amato M B P, Arnold J H, Bayford R, Bodenstein M, Böhm S H, Brown B H, Frerichs I, Stenqvist O, Weiler N \& Wolf G K 2012 Physiological Measurement 33(5), 679-694.

Adler A, Amyot R, Guardo R, Bates J H \& Berthiaume Y 1997 Journal of applied physiology (Bethesda, Md. : 1985) 83(5), 1762-7.

Adler A, Arnold J H, Bayford R, Borsic A, Brown B H, Dixon P, Faes T J C, Frerichs I, Gagnon H, Gärber Y, Grychtol B, Hahn G, Lionheart W R B, Malik A, Patterson R P, Stocks J, Tizzard A, Weiler N \& Wolf G K 2009 Physiological measurement 30(6), S35-55.

Adler A, Guardo R \& Berthiaume Y 1996 IEEE transactions on bio-medical engineering 43(4), 414-20.

Barber D C \& Seagar A D 1987 Clinical Physics and Physiological Measurement 8(4A), 47-54.

Brown B H, Barber D C \& Seagar a D 1985 Clinical physics and physiological measurement : an official journal of the Hospital Physicists' Association, Deutsche Gesellschaft für Medizinische Physik and the European Federation of Organisations for Medical Physics 6(2), 109-21.

Brown B H \& Seagar A D 1987 Clinical Physics and Physiological Measurement 8(4A), 91-97.

Cheney M, Isaacson D, Newell J C, Simske S \& Goble J 1990 International Journal of Imaging Systems and Technology 2(2), 66-75.

Frerichs I, Dargaville P A, Dudykevych T \& Rimensberger P C 2003 Intensive care medicine 29(12), 2312-6.

Gabriel C, Gabriel S \& Corthout E 1996 Physics in medicine and biology 41(11), 2231-49.

Gabriel C, Peyman A \& Grant E H 2009 Physics in medicine and biology 54(16), 4863-78. 
Gabriel S, Lau R \& Gabriel C 1996 Physics in Medicine and Biology 41(11), 2251-2269.

Grychtol B \& Adler A 2013 Physiological measurement 34(6), 579-93.

Grychtol B, Lionheart W R B, Wolf G K, Bodenstein M \& Adler A 2012 IEEE transactions on medical imaging 31(9), 1754-1760.

Grychtol B, Wolf G K, Adler A \& Arnold J H 2010 Physiological measurement 31(8), S31-43.

Lesparre N, Gibert D, Nicollin F, Nussbaum C \& Adler A 2013 Geophysical Journal International 195(2), 972-984.

Lionheart W R B 2004 Physiological Measurement 25(1), 125-142.

Nopp P, Harris N D, Zhao T X \& Brown B H 1997 Medical \& biological engineering \& computing 35(6), 695-702.

Pelosi P, D'Andrea L, Vitale G, Pesenti A, Gattinoni L \& Andrea L D 1994 American journal of respiratory and critical care medicine 149(1), 8-13.

Polydorides N \& Lionheart W R B 2002 Measurement Science and Technology 13(12), 1871-1883.

Schöberl J 1997 Computing and Visualization in Science 1(1), 41-52.

Shaw G, Goussard Y \& Guardo R 1993 in 'Proceedings of the 15th Annual International Conference of the IEEE Engineering in Medicine and Biology Society' IEEE pp. 82-83.

Victorino J A, Borges J a B, Okamoto V N, Matos G F J, Tucci M R, Caramez M P R, Tanaka H, Sipmann F S, Santos D C B, Barbas C S V, Carvalho C R R \& Amato M B P 2004 American journal of respiratory and critical care medicine 169(7), 791-800.

Wolf G K, Gómez-Laberge C, Rettig J S, Vargas S O, Smallwood C D, Prabhu S P, Vitali S H, Zurakowski D \& Arnold J H 2013 Critical care medicine 41(5), 1296-304. 